\title{
Impact of Various Signal Detection Schemes on Performance Assessment of DCT-IFDMA SC-FDMA System
}

\author{
Md. Afzal Hussain ${ }^{1}$, Mahmudul Haque Kafi ${ }^{2}$, Sk. Sifatul Islam ${ }^{2}$ and Shaikh \\ Enayet ullah ${ }^{3}$ \\ ${ }^{1}$ Spectrum Engineering Consortium Ltd., Dhaka-1205, Bangladesh \\ ${ }^{2}$ Postgraduate student, Department of Applied Physics and Electronic \\ Engineering \\ Rajshahi-6205, Bangladesh \\ ${ }^{3}$ Department of Applied Physics and Electronic Engineering, University of \\ Rajshahi, Rajshahi-6205, Bangladesh \\ emon4950@yahoo.com,mahmudkafi49@gmail.com,chisty56@gmail.comand \\ enayet67@yahoo.com
}

\begin{abstract}
In this paper, an effort has been made to observe critically the impact of various channel coding and signal detection schemes in DCT-IFDMA aided SC-FDMA wireless communication system on color image transmission. The simulated system incorporates MMSE-SIC , OSIC, ZF and MMSE as signal detection and $1 / 2$-rated irregular LDPC and Repeat and Accumulate as channel coding schemes under $4 \times 4$ antenna configuration. It is noticeable from MATLAB based simulative study that the system shows quite satisfactory performance in retrieving transmitted color image under scenario of hostile fading channel environment with implementation of MMSE-SIC signal detection, QAM digital modulation and Repeat and Accumulate channel coding scheme.
\end{abstract}

Keywords: DCT, IFDMA, SC-FDMA, Signal to noise ratio(SNR), MMSE-SIC , OSIC, $Z F$ and $M M S E$

\section{Introduction}

With rapid increase in the demand of ubiquitous communication services, it is observable in recent years a massive deployment of cellular networks. Several cellular technologies have surfaced over the past some time and the long term evolution (LTE) and its successor LTE-advanced (LTE-A) networks have already been deployed commercially in different regions of the world and attracted considerable attention in the cellular domain. The LTE-A networks use single-carrier frequency division multiple access (SC-FDMA) for uplink transmissions.

In comparison to OFDMA, the SC-FDMA significantly reduces the envelope fluctuations in the transmitted waveform. The SC-FDMA signals have inherently lower peak-to-average power ratio (PAPR) than the OFDMA signals. As the SC-FDMA system uses different sub channels(subsets of subcarriers) to transmit the information symbols in parallel with each other, various subcarriers assigning schemes such as IFDMA( Interleaved FDMA), DFDMA(Distributed FDMA) and LFDMA (Localized FDMA) are used in SC-FDMA systems. The SC-FDMA systems with IFDMA and LFDMA have a better PAPR performance than OFDMA systems. In Device-to-device (D2D) communication-enabled cellular networks, cellular devices are allowed to directly communicate with each other without any evolved NodeB (eNB). D2D communication aims to improve the spectral efficiency and increases the overall system capacity. In 2015, the SC-FDMA has been adopted as UL transmission scheme using fractional frequency 
reuse (FFR)-based architecture to efficiently The proposed scheme uses fractional frequency reuse (FFR)-based architecture to efficiently allocate the resources and mitigate the interference between CUEs(conventional cellular user equipment) and D2D user equipment (DUEs)[1,2]. In 2013, Elbehery and et.al.,made a performance evaluative study for Continuous Phase Modulation (CPM) aided Discrete Cosine Transform based Single Carrier Frequency Division Multiple Access (DCT-SC-FDMA) system on image transmission. The study ratified that such system outperformed as compared to conventional QPSK based SC-FDMA system with achievement of higher Peak Signal to Noise Ratio (PSNR) values [3].

In this present study, an effort has been made to observe the performance of DCTIFDMA SC-FDMA System on color image transmission.

\section{Signal Processing Techniques}

We assume that the color image is pre-processed through various schemes prior to transmission through a $4 \times 4$ MIMO fading channel. The received signal Yin terms of channel matrix $\mathrm{H}$, transmitted signal $\mathbf{X}$ and additive white Gaussian noise(AWGN) $\mathrm{N}$ with a variance of ${\sigma_{\mathbf{n}}}^{2}$ can be written as

$$
\mathrm{Y}=\mathrm{HX}+\mathrm{N}
$$

In our present study various signal processing schemes have been used. A brief overview of these schemes is given below:

\subsection{Zero-Forcing $(\mathrm{ZF})$}

In Zero-Forcing (ZF) signal detection scheme, the ZF weight matrix is given by

$$
W_{Z F}=\left(H^{H} H\right)^{-1} H^{H}
$$

and the detected desired signal $\tilde{\mathbf{X}}_{\mathbf{Z F}}$ from the transmitting antenna is given by

$$
\tilde{X}_{Z F}=W_{Z F} Y
$$

\subsection{Minimum Mean Square Error (MMSE)}

In Minimum mean square error (MMSE) based signal detection scheme, the MMSE weight matrix is given by

$$
\mathrm{W}_{\text {MMSE }}=\left(\mathrm{H}^{\mathrm{H}} \mathrm{H}+\sigma_{\mathrm{n}}{ }^{2} \mathrm{I}\right)^{-1} \mathrm{H}^{\mathrm{H}}
$$

Where $(.)^{\mathrm{H}}$ denotes the Hermitian transpose operation and the detected desired signal $\tilde{\mathbf{X}}_{\text {MMSE }}$ from the transmitting antenna is given by

$$
\tilde{\mathrm{X}}_{\text {MMSE }}=\mathrm{W}_{\text {MMSE }}{ }^{\mathrm{Y}}
$$

\subsection{Ordered Successive Interference Cancellation (OSIC)}

In Ordered successive interference cancellation (OSIC) signal detection scheme, its implementation is performed in four steps. In first step, the first detected signal/data stream $\tilde{\mathbf{X}}_{\text {OSIC-1 }}$ and modified form of received signal $\tilde{\mathbf{Y}}_{\text {OSIC }-1}$ can be written as:

$$
\tilde{\mathbf{X}}_{\text {OSIC-1 }}=\mathbf{W}_{(\operatorname{MMSE}(1, ;)} \mathbf{Y}
$$


$\tilde{\mathbf{Y}}_{\text {OSIC }-1}=\mathbf{Y}-\mathbf{H}_{(:, 1)} \tilde{\mathbf{X}}_{\text {OSIC }-1}$

In second step, the second detected signal/data stream $\tilde{\mathbf{X}}_{\text {OSIC-2 }}$ and modified form of received signal $\tilde{\mathbf{Y}}_{\text {OSIC-2 }}$ can be written as:

$\tilde{\mathbf{X}}_{\text {OSIC-2 }}=\mathbf{W}_{(\mathbf{M M S E}(2,:)} \tilde{\mathbf{Y}}_{\text {OSIC-1 }}$

$\tilde{\mathbf{Y}}_{\text {OSIC }-2}=\tilde{\mathbf{Y}}_{\text {OSIC }-1}-\mathbf{H}_{(:, 2)} \tilde{\mathbf{X}}_{\text {OSIC }-2}$

In third step, the third detected signal/data stream $\tilde{\mathbf{X}}_{\text {OSIC-3 }}$ and modified form of received signal $\tilde{\mathbf{Y}}_{\text {OSIC-3 }}$ can be written as:

$\tilde{\mathbf{X}}_{\text {OSIC-3 }}=\mathbf{W}_{(\mathbf{M M S E}(3, ;)} \tilde{\mathbf{Y}}_{\text {OSIC-2 }}$
$\tilde{\mathbf{Y}}_{\text {OSIC-3 }}=\tilde{\mathbf{Y}}_{\text {OSIC-2 }}-\mathbf{H}_{(: 3)} \tilde{\mathbf{X}}_{\text {OSIC-3 }}$

In fourth step, the fourth detected signal/data stream $\tilde{\mathbf{X}}_{\text {OSIC-4 }}$ and modified form of received signal $\tilde{\mathbf{Y}}_{\text {OSIC-4 }}$ can be written as:

$\tilde{\mathbf{X}}_{\text {OSIC-4 }}=\mathbf{W}_{(\mathbf{M M S E}(4,:)} \tilde{\mathbf{Y}}_{\text {OSIC-3 }}$
$\tilde{\mathbf{Y}}_{\text {OSIC-4 }}=\tilde{\mathbf{Y}}_{\text {OSIC-3 }}-\mathbf{H}_{(:, 4)} \tilde{\mathbf{X}}_{\text {OSIC-4 }}$

where, $\mathbf{W}_{\text {(MMSE(1,:) }}, \mathbf{W}_{(\mathbf{M M S E}(2, ;)}, \mathbf{W}_{(\mathbf{M M S E}(3,))}$ and $\mathbf{W}_{(\mathbf{M M S E}(4, ;)}$ are the first, second, third and fourth rows of MMSE weight matrix and $\mathrm{H}_{(:, 1)}, \mathrm{H}_{(:, 2)}, \mathrm{H}_{(: 3)}$, and $\mathrm{H}_{(:, 4),:)}$ are the first, second, third and fourth columns of the channel matrix respectively. The detected desired signal $\tilde{\mathbf{x}}_{\text {OSIC }} \in \mathrm{C}^{4 \times 4562325}$ from the transmitting antenna is given by[2,4]

\subsection{Repeat and Accumulate (RA)}

In RA, a powerful modern error-correcting coding scheme, the extracted binary bits from the color image is rearranged into blocks with each block containing 2048 binary bits. The binary bits in each block is repeated 2 times and permuted by an interleaver of length 4096. The interleaved binary data block $\mathrm{z}$ is passed through a truncated rate-1 twostate convolutional encoder whose output $\mathrm{x}$ is the Repeat and Accumulate encoded binary data and is given by $\mathrm{x}=\mathrm{zG}$, where $\mathrm{G}$ is an $4096 \times 4096$ matrix with 1 s on and above its main diagonal and 0s elsewhere[5]. The RA encoded blocked binary data are further processed to produce a $442368 \times 1$ single column vector data.

\subsection{Low Density Parity-check Matrix (LDPC)}

In LDPC coding, 1/2.-rated irregular LDPC code is used with a code length of 1024 bits. Its parity-check matrix $[\mathrm{H}]$ is a sparse matrix with a dimension of $512 \times 1024$ and contains only three 1's in each column and six 1's in each row. The parity-check matrix $[\mathrm{H}]$ is formed from a concatenation of two matrices $[\mathrm{A}]$ and $[\mathrm{P}]([\mathrm{H}]=[\mathrm{A}] \mid[\mathrm{P}])$, each has a dimension of $512 \times 512$ ). The columns of the parity-check matrix $[\mathrm{H}]$ is rearranged to produce a new parity-check matrix $[\mathrm{newH}]$. With rearranged matrix elements, the matrix [A] becomes non-singular and it is further processed to undergo LU decomposition. The parity bits sequence [p] is considered to have been produced from 
a block based input binary data sequence $[\mathbf{u}]=\left[\mathrm{u}_{1} \mathrm{u}_{2} \mathrm{u}_{3} \mathrm{u}_{4} \ldots \ldots . \mathrm{u}_{512}\right]^{\mathrm{T}}$ and three matrices $[\mathrm{P}]($ of $[$ newH]), $[\mathrm{L}]$ and $[\mathrm{U}]$ using the following Matlab notation :

$\mathbf{p}=\bmod (\mathrm{U} \backslash(\mathrm{Llz}), 2) ;$ where, $\mathrm{z}=\bmod \left(\mathrm{P}^{*} \mathrm{u}, 2\right)$;

The LDPC encoded $1024 \times 1$ sized block based binary data sequence [c] is formulated from concatenation of parity check bit $\mathbf{p}$ and information bit $\mathbf{u}$ as :

\section{$[\mathbf{c}]=[\mathbf{p} ; \mathbf{u}]$}

The first 512 bits of the codeword matrix [c] are the parity bits and the last 512 bits are the information bits. In iterative Log Domain Sum-Product LDPC decoding Algorithm, the transmitted bits are retrieved [6-7].

\subsection{Minimum Mean Square Error Successive Interference Cancellation (MMSE-} SIC)

In Minimum mean square error successive interference cancellation (MMSE-SIC) scheme, the extended channel matrix $\overline{\mathrm{H}}$ and the extended received signal $\overline{\mathrm{Y}}$ in terms of identity and null matrices are given by

$$
\begin{aligned}
& \overline{\mathrm{H}}=\left[\begin{array}{l}
H \\
\left(\sqrt{\sigma^{2}{ }_{n}}\right) I
\end{array}\right] \\
& \overline{\mathrm{Y}}=\left[\begin{array}{l}
\mathrm{Y} \\
\mathbf{0}
\end{array}\right]
\end{aligned}
$$

On QR decomposition of $\overline{\mathrm{H}}$, an orthogonal matrix $\overline{\mathrm{Q}}$ and upper triangular matrix $\overline{\mathrm{R}}$ are produced. Equation (9) is multiplied with $\overline{\mathrm{Q}}^{\mathrm{T}}$ to provide a modified form of received signal $\overline{\bar{Y}}$ with neglected noise component

$$
\overline{\bar{Y}}=\overline{\mathrm{Q}}^{\mathrm{T}} \overline{\mathrm{Y}}=\overline{\mathrm{Q}}^{\mathrm{T}} \overline{\mathrm{H}} \mathrm{X}=\overline{\overline{\mathrm{R}}} \mathrm{X}
$$

Considering a single time slot, the transmitted four signals $\overline{\bar{X}}_{1}, \overline{\bar{X}}_{2}, \overline{\bar{X}}_{3}$ and $\overline{\bar{X}}_{4}$ in terms of four received signals $\overline{\bar{Y}}_{1}, \overline{\bar{Y}}_{2}, \overline{\bar{Y}}_{3}$ and $\overline{\bar{Y}}_{4}$ ( First through Fourth rows of $\overline{\bar{Y}}$ and neglecting otherrow data) and the components of matrix $\overline{\overline{\mathrm{R}}}$ in first through fourth row) can be obtained from a matrix equation as[8]:

$$
\overline{\overline{\mathrm{Y}}}[(:, 1)]=\left[\begin{array}{c}
\overline{\overline{\mathrm{Y}}}_{1} \\
\overline{\overline{\mathrm{Y}}}_{2} \\
\overline{\overline{\mathrm{Y}}}_{3} \\
\overline{\overline{\mathrm{Y}}}_{4} \\
\overline{\overline{\mathrm{Y}}}_{5} \\
\overline{\overline{\mathrm{Y}}}_{6} \\
\overline{\overline{\mathrm{Y}}}_{7} \\
\overline{\overline{\mathrm{Y}}}_{8}
\end{array}\right]=\left[\begin{array}{cccc}
\overline{\overline{\mathrm{R}}}_{1,1} & \overline{\overline{\mathrm{R}}}_{1,2} & \overline{\overline{\mathrm{R}}}_{1,3} \overline{\overline{\mathrm{R}}}_{1,4} \\
0 & \overline{\overline{\mathrm{R}}}_{2,2} & \overline{\overline{\mathrm{R}}}_{2,3} & \overline{\overline{\mathrm{R}}}_{2,4} \\
0 & 0 & \overline{\overline{\mathrm{R}}}_{3,3} & \overline{\overline{\mathrm{R}}}_{3,4} \\
0 & 0 & 0 & \overline{\overline{\mathrm{R}}}_{4,4} \\
0 & 0 & 0 & 0 \\
0 & 0 & 0 & 0 \\
0 & 0 & 0 & 0 \\
0 & 0 & 0 & 0
\end{array}\right]\left[\begin{array}{c}
\overline{\overline{\mathrm{X}}}_{1} \\
\overline{\overline{\mathrm{X}}}_{2} \\
\overline{\overline{\mathrm{X}}}_{3} \\
\overline{\overline{\mathrm{X}}}_{4}
\end{array}\right]
$$




\subsection{2-D Median Filtering}

In 2-D Median Filtering scheme, a $3 \times 3$ neighborhood windowing mask is used for simply sorting all the pixel values within the window and finding the median value and replacing the original pixel value with the median value[9].

\section{System Description}

A RGB color image with 96 pixels (width) $\times 96$ pixels(height) is processed in a SCFDMA system depicted in Figure 1.The color image is converted into their respective three Red, Green and Blue components with each component is of 96 pixels $\times 96$ pixels in size The pixel integer values[0-255] are converted into 8 bits binary form and channel coded and interleaved and digitally modulated using QAM and QPSK . The complex symbols are blocked with each block consisting of 128 symbols. A 128 point discrete cosine transformation (DCT) algorithm is applied to each block. An interleaved subcarrier mapping scheme is applied to each block making the size of each block 512 viz. three zeros are inserted between two consecutive symbols. On application of 512 point inverse DCT, a transformed data of size $512 \times 1728$ symbols. On cyclic prefixing, its size is $532 \times 1728$ symbols. The data is multiplexed into 4 streams. The first stream consists of data of 1 st column, 5th column, 9th column and so on. The second stream consists of data of 2nd column, 6th column, 10th column and so on. The third stream consists of data of 3rd column, 7th column, 11th column and so on. The fourth stream consists of data of 4th column, 8th column, 12th column and so on. Eventually, data are transmitted from four dual polarized antennas. In receiving end, transmitted signals are detected using various signal detection techniques. The detected signals are demultiplexed with subsequent cyclic prefix removing,512 point DCT transformed, subcarrier demapped, 128 point inverse DCT transformed, demodulated, deinterleaved, channel decoded and eventually retrival of color image is made [10-11].

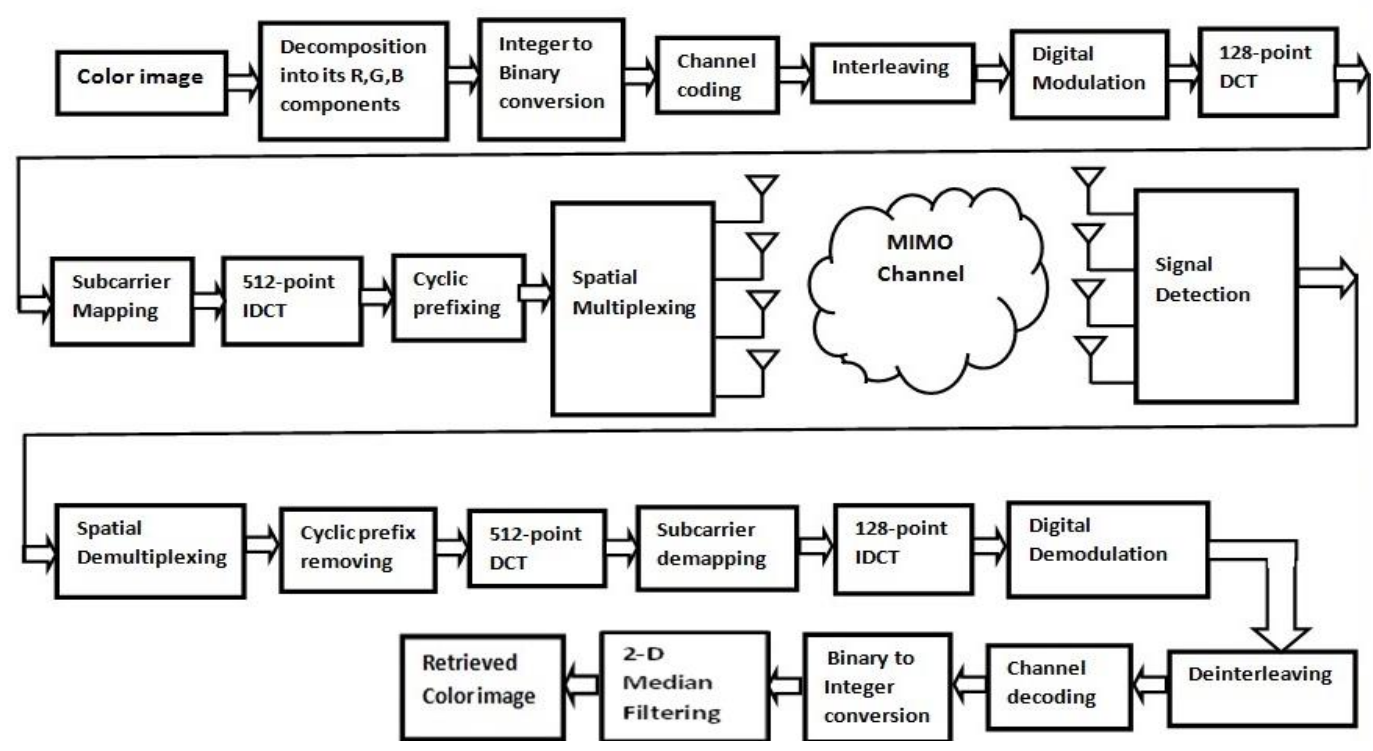

Figure 1. Block Diagram of DCT-IFDMA SC-FDMA System

\section{Result and Discussion}

In this section, we have presented a series of simulation results to illustrate the significant impact of system performance in terms of BER in a DCT-IFDMA SC-FDMA System with simulation parameters tabulated in Table 1. 
Table 1. Summary of the Simulated Model Parameters

\begin{tabular}{|c|c|}
\hline Data type & Color image (96x96x3 pixels) \\
\hline $\begin{array}{c}\text { No of interleaved sub carrier mapped complex } \\
\text { symbols used in inverse DCT transformation }\end{array}$ & 512 \\
\hline $\begin{array}{c}\text { No of complex modulated symbols used in DCT } \\
\text { transformation }\end{array}$ & 128 \\
\hline bandwidth spreading factor in IFDMA & 20 symbols \\
\hline Cyclic prefixing & Repeat and Accumulate Code and LDPC \\
\hline Channel Coding & Log-domain sum product with 5 iterations \\
\hline LDPC decoding algorithm & $4 \times 4$ \\
\hline Antenna configuration with dual polarization & QPSK and QAM \\
\hline Data Modulation & MMSE-SIC,OSIC, ZF and MMSE \\
\hline Signal detection Scheme & AWGN and Rayleigh fading \\
\hline Channel & 0 to 5 dB \\
\hline Signal to noise ratio, SNR & \\
\hline
\end{tabular}

On critical observation of graphical illustrations presented in Figure 2 through Figure 5, it is quite evident that the DCT-IFDMA SC-FDMA system shows comparatively satisfactory performance with QAM digital modulation and Repeat and Accumulate channel coding scheme over a significant part of SNR values. In all cases, the system performance is well defined under scenario of implementing various signal detection, digital modulation and channel coding and without utilization of 2-D median image filtering schemes. In Figure 2 of MMSE-SIC signal detection implemented system, the estimated BER values are 0.1707 and 0.0841 in a typically assumed SNR value of $1 \mathrm{~dB}$ for QPSK digital modulation with LDPC channel coding as compared to QAM digital modulation with Repeat and Accumulate channel coding which is indicative of a system performance improvement of $3.07 \mathrm{~dB}$. In case of identical signal and noise power( (0dB), the estimated BER value under utilization of MMSE-SIC, QAM and Repeat and accumulate channel coding is merely 9.13\%. In Figure 3 for OSIC implemented system, it is observable that the system shows satisfactory performance in QAM with Repeat and accumulate channel coding and worst performance in QPSK with LDPC channel coding. Under such case, the estimated BER values at $1 \mathrm{~dB}$ SNR values are found to be of 0.0889 and 0.1843 which ratifies a $3.17 \mathrm{~dB}$ system performance improvement. At $10 \%$ BER, a SNR improvement of $1.25 \mathrm{~dB}$ is found in

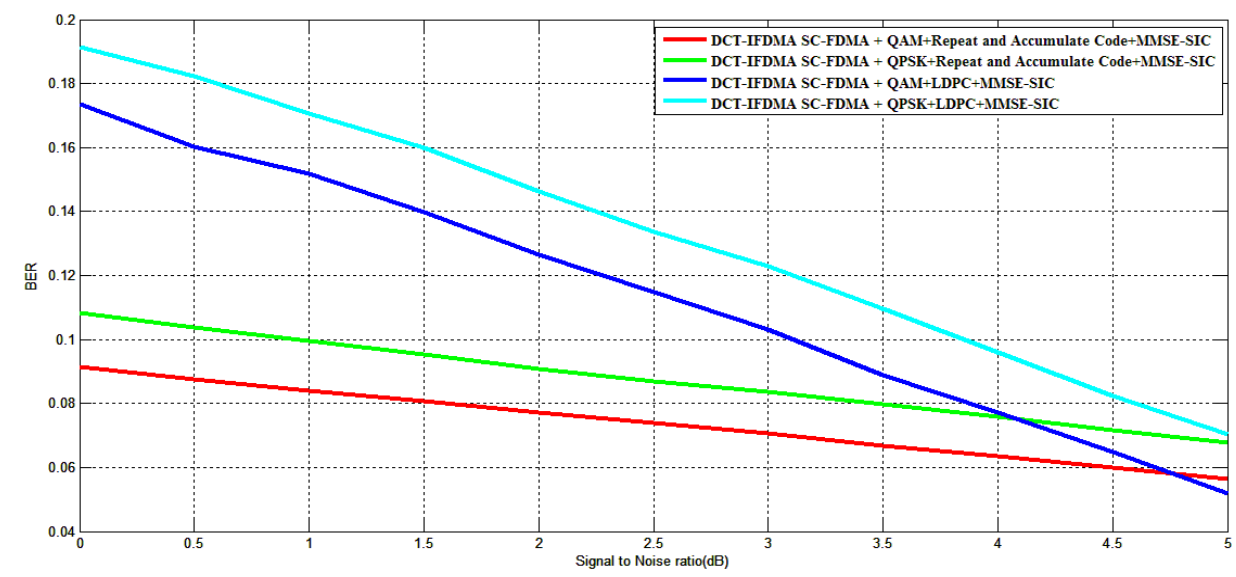

Figure 2. BER performance comparison of dual polarized DCT-IFDMA aided SC-FDMA wireless communication system under various digital modulations and MMSE-SIC signal detection scheme 
QAM with LDPC as compared to QPSK with LDPC. In Figure 4 for ZF implemented

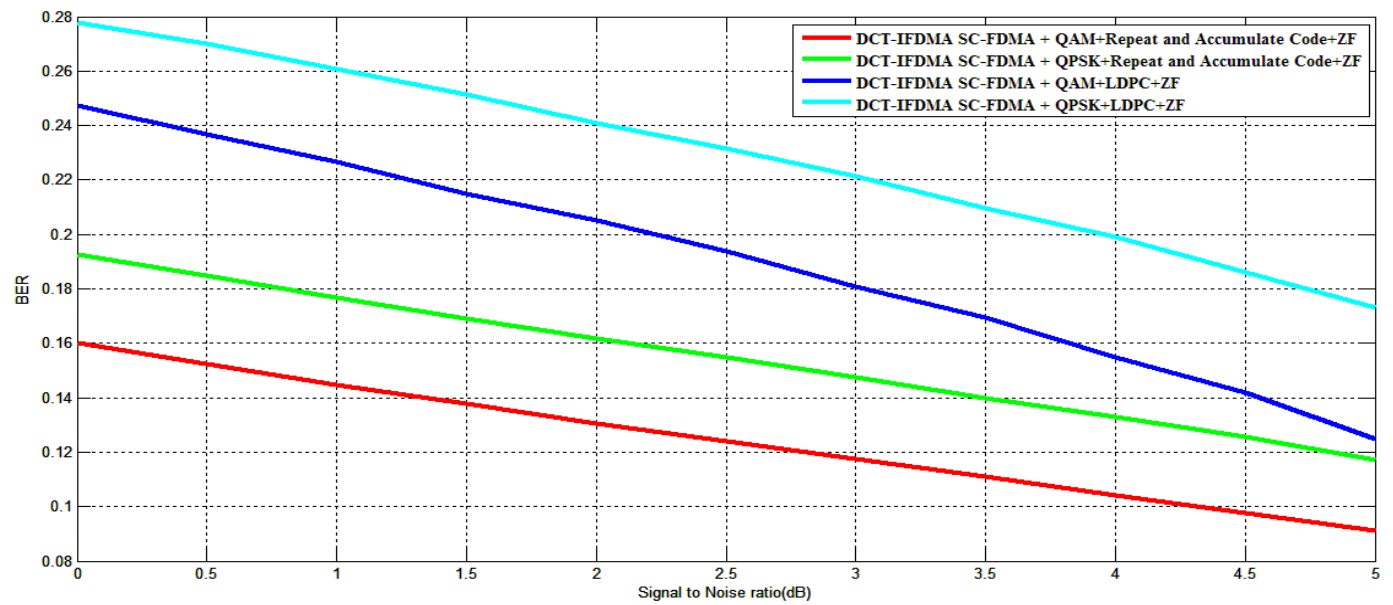

Figure 3. BER performance comparison of DCT-IFDMA aided SC-FDMA wireless communication system under various digital modulations and OSIC signal detection scheme

System, it shows satisfactory performance in QAM with Repeat and accumulates channel coding and worst performance in QPSK with LDPC channel coding. In such case, the estimated BER values are 0.1449 and 0.2608 for a typically assumed SNR value of $1 \mathrm{~dB}$ which indicates a system performance improvement of 2.55dB.In Figure 5 for MMSE implemented system, it is noticeable that the system shows satisfactory performance in

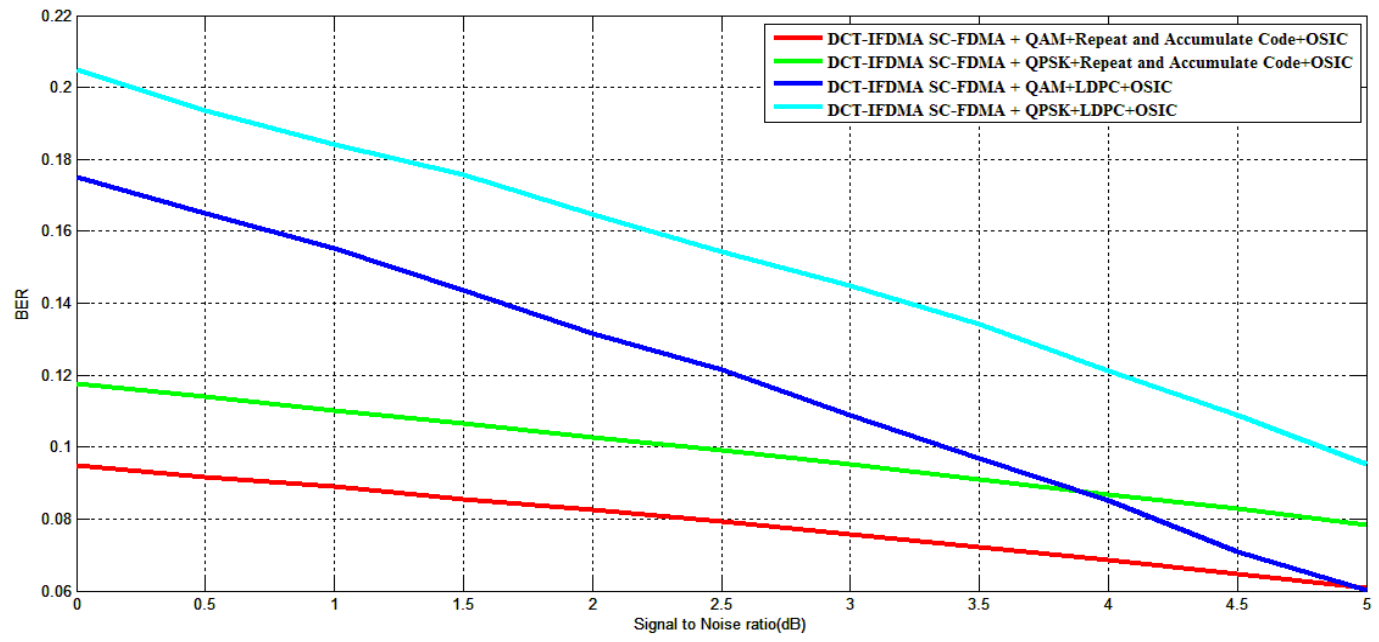

Figure 4. BER performance comparison of DCT-IFDMA aided SC-FDMA wireless communication system under various digital modulations and ZF signal detection scheme 
QAM with Repeat and accumulate channel coding and worst performance in QPSK with LDPC channel coding. The estimated BER values are 0.0849 and 0.1721 for a typically assumed SNR value of $1 \mathrm{~dB}$ which indicates a system performance improvement of $3.07 \mathrm{~dB}$. On careful analysis of all presented simulation results, the simulated system is found to have shown most satisfactory performance in specific case of utilizing MMSESIC signal detection, Repeat \& Accumulate channel coding and QAM digital modulation. In Figure 6, the transmitted and retrieved color images have been presented with implementation of MMSE-SIC signal detection, QAM digital modulation, Repeat and Accumulate channel coding and 2-D median image filtering.

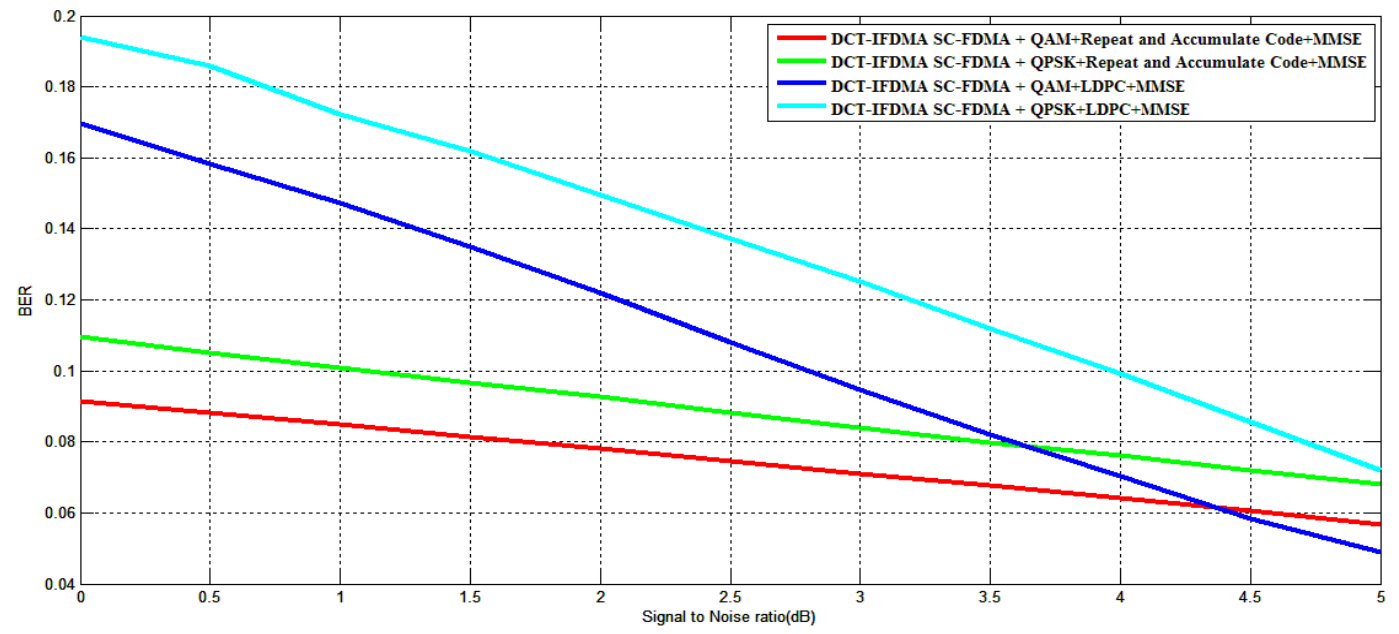

Figure 5. BER performance comparison of DCT-IFDMA aided SC-FDMA wireless communication system under various digital modulations and MMSE signal detection scheme
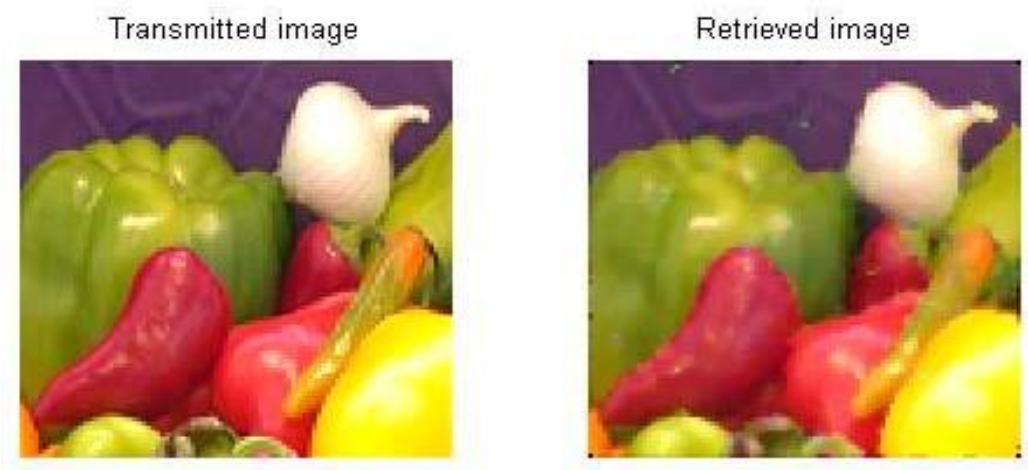

\section{Figure 6. Performance indicator of DCT-IFDMA aided SC-FDMA wireless communication system under implementation of MMSE-SIC signal detection, QAM digital modulation and 2-D median image filtering for a typical image at SNR value of $5 \mathrm{~dB}$}

\section{Conclusions}

In this paper, the performance of DCT-IFDMA aided SC-FDMA wireless communication system has been investigated on color image transmission using various signal detection and FEC channel encoded schemes. The results show that the implementation of MMSE-SIC signal detection scheme with Repeat and Accumulate channel coding and QAM digital modulation ratifies the robustness of system 
performance in retrieving color image transmitted over Gaussian noise contaminated and Rayleigh fading channels. Such system can be utilized for other form of data transmission in hostile fading channels where induced noise with power is comparable with signal power.

\section{References}

[1] S. T. Shah, J. Gu, S. F. Hasan, and M. Y. Chung, "2015: SC-FDMA-based resource allocation and power control scheme for D2D communication using LTE-A uplink resource", EURASIP Journal on Wireless Communications and Networking, vol. 137, pp. 1-15

[2] Y. S. Cho, J. Kim, W. Y. Yang and C. G. Kang, 2010: MIMO-OFDM Wireless Communications with MATLAB, John Wiley and Sons (Asia) PTE Limited, Singapore.

[3] A. Elbehery, S. A. S. Abdelwahab, M. Abd El Naby, E. S. Hassan and S. Elaraby, "2013: Image Transmission with DCT Based SC-FDMA System Using Continuous Phase Modulation”, paper presented in 30th National Radio Science Conference, Egypt.

[4] S. Hosain, M. Haque and S. E. Ullah, "2015: Performance Assessment of MimoMccdma System on Video Signal Transmission with Implementation of Various Digital Signal Processing Techniques'”, International Journal of Wireless Communications and Mobile Computing (WCMC), vol. 3, no. 2 , pp. 18-26, Science Publishing Group, New York, USA

[5] G. M. Vitetta, D. P. Taylor, G. Colavolpe, F. Pancaldi and P. A. Martin, "2013: Wireless Communications Algorithmic Techniques", John Wiley and Sons Ltd, United Kingdom.

[6] C. B. Schlegel and L. C. Perez, "2004: Trellis and turbo coding", John Wiley and Sons, Inc., publication, Canada.

[7] Bagawan Sewu Nugroho, https://sites.google.com/site/bsnugroho/ldpc

[8] L. Bai and J. Choi, "2012: Low Complexity MIMO Detection, Springer Science and Business Media", LLC, New York, USA.

[9] O. Marques, "Practical Image and Video Processing Using MATLAB", John Wiley and Sons, New Jersey, USA, (2011).

[10] F. E. A. El-Samie, F. S. Al-kamali, A. Y. Al-nahari and M. I. Dessouky, "2013: SC-FDMA for Mobile Communications", CRC Press, Taylor \& Francis Group, Florida, USA.

[11] A. Goldsmith, "Wireless Communications", 2005, First Edition, Cambridge University Press, United Kingdom.

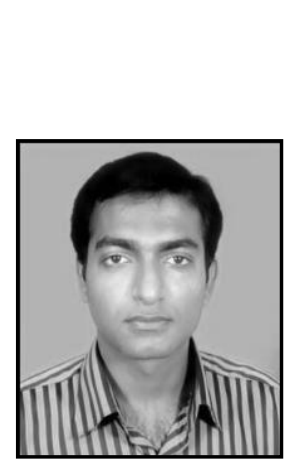

\section{Authors}

Md. Afzal Hussain is working as a Network Engineer in Presales/ Solution Department, Spectrum Engineering Consortium Ltd.. He received her B.Sc. (Hons.) and M.Sc. degree both in Applied Physics and Electronic Engineering department from university of Rajshahi in 2011 and 2012 respectively. He is currently working on Configuring Routers, Access Points, Wireless routers, VPN routers, Wireless Controller, Layer 2 \& Layer 3 switches, IP camera, Network Access Storage (NAS) using D-Link. His research interest is developing and implementing new beam forming algorithm for effective wireless linking between computers in data centers.

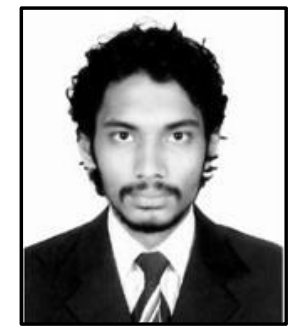

Mahmudul Haque Kafi received his B.Sc.(Hons) and M.Sc. degree in Applied Physics and Electronic Engineering from University of Rajshahi in 2013 and 2014 respectively. Concurrently, he is engaged with simulation study on MIMO wireless communication. His research interest is in Transmit/Receive antenna diversity, Channel coding, MIMOOFDM, MGSTC, mmWave system in Audio, text and Video (one dimensional and multiview) signal transmission in hostile channels and their effective retrieval. 


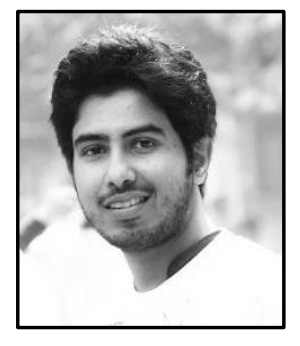

Sk. Shifatul Islam did his B.Sc.(Hons) in Applied Physics and Electronic Engineering from University of Rajshahi in 2013. Concurrently, he is doing his M.Sc. from the same department and doing his M.SC Thesis entitled Video signal transmission in single user $5 \mathrm{~g}$ compatible hybrid precoded millimeter wave wireless communication system. His research interest is in Transmit/Receive antenna diversity, Channel coding, MIMOOFDM, MGSTC, mmWave system in Audio, text and Video (one dimensional and multiview) signal transmission in hostile channels and their effective retrieval.

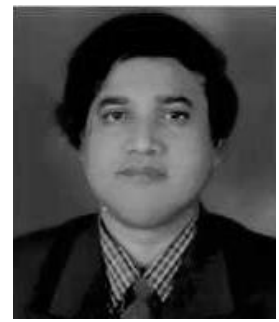

Shaikh Enayet Ullah is a Professor of the Department of Applied Physics and Electronic Engineering, Faculty of Engineering, University of Rajshahi, Bangladesh. He received US equivalent B.Sc. and M.Sc. degree in Physics and Electronics and Ph.D. degree in Physics in 1983, 1985 and 2000 respectively. He worked as a Professor and Chairman (on deputation) in the Department of Information and Communication Engineering, University of Rajshahi. He is working as a member of both Editorial and Reviewer Board of various International Journals. He has published more than 70 papers in multidisciplinary fields. His main research interests include Cooperative communications, MIMO-OFDM, WiMAX and LTE-Advanced and Potential Field geophysical data inversion 